\title{
ON IMAGES AND INVERSE IMAGES OF WEIERSTRASS POINTS $\dagger$
}

\author{
by R. F. LAX
}

(Received 29 November, 1976)

1. Introduction. The classical theory of Weierstrass points on a compact Riemann surface is well-known (see, for example, [3]). Ogawa [6] has defined generalized Weierstrass points. Let $Y$ denote a compact complex manifold of (complex) dimension $n$. Let $E$ denote a holomorphic vector bundle on $Y$ of rank $q$. Let $J^{k}(E)(k=0,1, \ldots)$ denote the holomorphic vector bundle of $k$-jets of $E\left[2\right.$, p. 112]. Put $r_{k}(E)=\operatorname{rank} J^{k}(E)=$ $q .(n+k) ! / n ! k$ !. Suppose that $\Gamma(E)$, the vector space of global holomorphic sections of $E$, is of dimension $\gamma(E)>0$. Consider the trivial bundle $Y \times \Gamma(E)$ and the map

$$
j_{k}=j_{k}^{E}: Y \times \Gamma(E) \rightarrow J^{k}(E),
$$

which at a point $Q \in Y$ takes a section of $E$ to its $k$-jet at $Q$. Put $\mu=\min \left(\gamma(E), r_{k}(E)\right)$.

Definition ([6]). Let $W_{k}(E)$ denote the reduced closed analytic subspace of $Y$ defined by the vanishing of the exterior power $\Lambda^{\mu} j_{k}$. We call $W_{k}(E)$ the space of $k$ th order Weierstrass points of $E$.

Note that if $r_{k}(E) \leq \gamma(E)$, then the points of $W_{k}(E)$ are those $Q \in Y$ such that the map $j_{k, Q}: \Gamma(E) \rightarrow J_{Q}^{k}(E)$ is not surjective and that if $r_{k}(E) \geq \gamma(E)$, then the points of $W_{k}(E)$ are those $Q \in Y$ such that $j_{k, Q}$ is not injective.

Suppose that $X$ is a compact complex manifold and $f: X \rightarrow Y$ is a holomorphic map. Let $P \in X$ and put $Q=f(P)$. Then we have the following commutative diagram of $\mathbb{C}$-linear maps.

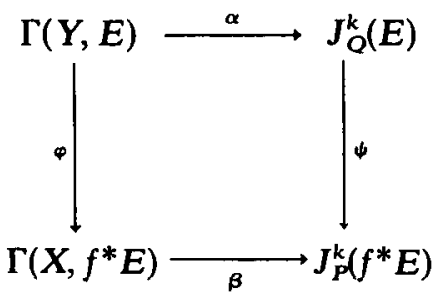

Here $\alpha$ and $\beta$ are the maps $j_{k, Q}^{E}$ and $j_{k, P}^{j^{*} E}$ respectively, and $\varphi$ and $\psi$ are induced by composition with $f$ (cf. [2, p. 113]). We will use this diagram to show that under certain conditions on $f$ and $E$, we have $f\left(W_{k}\left(f^{*} E\right) \subseteq W_{k}(E)\right.$ or $f^{-1}\left(W_{k}(E)\right) \subseteq W_{k}\left(f^{*} E\right)$ as sets.

† This research was supported by NSF Grant MCS 76-06002.

Glasgow Math. J. 19 (1978) 125-128 


\section{An inverse image theorem.}

(2.1) THEOREM. With notation as in $\S 1$, suppose that the following three conditions are satisfied:

(i) $\gamma(E) \leq r_{k}(E)$,

(ii) $\gamma\left(f^{*} E\right) \leq r_{k}\left(f^{*} E\right)$,

(iii) $f$ is surjective.

Then $f^{-1}\left(W_{k}(E)\right) \subseteq W_{k}\left(f^{*} E\right)$ as sets.

Proof. Let $P \in X, Q=f(P)$ and suppose that $Q \in W_{k}(E)$. Since $f$ is surjective, the map $\varphi$ in diagram (*) is injective. Hence, if $\beta$ is injective, then $\alpha$ must be injective. But, since $Q \in W_{k}(E)$, the map $\alpha$ is not injective. Thus $\beta$ is not injective and $P \in W_{k}\left(f^{*} E\right)$.

(2.2) We may apply this theorem in the following situation. Let $Y$ be a compact Riemann surface of genus $g$ and put $X=Y^{n}$. Let $T_{Y}$ (resp. $T_{X}$ ) denote the complex analytic cotangent bundle on $Y$ (resp. on $X$ ). Then $\gamma\left(T_{Y}\right)=g$ and $\gamma\left(T_{X}\right)=$ $\frac{1}{2} \operatorname{dim} H_{1}(X, \mathbb{C})=n g$, using the Kunneth formula and standard facts about Kahler manifolds $[4$, p. 124].

Let $\pi_{i}: X \rightarrow Y$ denote projection on the $i$ th factor. It follows that we have an injection $0 \rightarrow \pi_{i}^{*} T_{Y} \rightarrow T_{X}$ and hence that $\gamma\left(\pi_{i}^{*} T_{Y}\right) \leq \gamma\left(T_{X}\right)=n g$. Suppose $g \geq 3$. Now, $\gamma\left(T_{Y}\right)=r_{g-1}\left(T_{Y}\right)$ and it is not hard to see that $\gamma\left(\pi_{i}^{*} T_{Y}\right) \leq n g \leq r_{g-1}\left(\pi_{i}^{*} T_{Y}\right)$. Hence, by our theorem,

$$
\pi_{i}^{-1}\left(W_{g-1}\left(T_{Y}\right)\right) \subseteq W_{g-1}\left(\pi_{i}^{*} T_{Y}\right) .
$$

Note that the points of $W_{g-1}\left(T_{Y}\right)$ are the classical Weierstrass points of $Y$. Also, since we have the injection $0 \rightarrow \pi_{i}^{*} T_{Y} \rightarrow T_{X}$, we obtain

$$
\pi_{i}^{-1}\left(W_{\mathrm{g}-1}\left(T_{\mathrm{Y}}\right)\right) \subseteq W_{\mathrm{g}-1}\left(T_{\mathrm{X}}\right)
$$

by arguing as in the proof of 2.1 and noting that $\gamma\left(T_{X}\right) \leq r_{g-1}\left(T_{X}\right)$.

(2.3) Remark. With notation as in 2.2 , we have shown in [5] that

$$
W_{8-1}\left(\Lambda^{n} T_{X}\right)=\bigcup_{i=1}^{n} \pi_{i}^{-1}\left(W_{8-1}\left(T_{Y}\right)\right)
$$

Thus, $W_{\mathrm{g}-1}\left(\Lambda^{n} T_{X}\right) \subseteq W_{\mathrm{g}-1}\left(T_{X}\right)$. Is there any reason for this relationship?

(2.4) We give an example here to show that the inverse image of a Weierstrass point is not always a Weierstrass point. Let $f: X \rightarrow Y$ be a smooth (i.e. unramified) 2-sheeted cover with both $X$ and $Y$ hyperelliptic, the latter of genus $g(\geq 2)$. Such a covering is explicitly constructed in [7, pp. 188 and 203]. By the Riemann-Hurwitz formula, $X$ has 
genus $2 g-1$. Let $T_{X}$ (resp. $T_{Y}$ ) denote the canonical bundle on $X$ (resp. on $Y$ ). Since $f$ is an unramified cover, it follows from $[1, \mathrm{VI}, 4.9]$ that $f^{*} T_{Y}=T_{X}$. Now, $W_{\mathrm{g}-1}\left(T_{Y}\right)$ consists of $2 g+2$ points, while $W_{g-1}\left(T_{X}\right) \subseteq W_{2 g-2}\left(T_{X}\right)$, which contains only $4 g$ points. Thus $f^{-1}\left(W_{g-1}\left(T_{Y}\right)\right)$ cannot be a subset of $W_{g-1}\left(T_{X}\right)$. Note that condition (ii) of 2.1 is violated.

\section{An image theorem.}

(3.1) THEOREM. With notation as in $§ 1$, suppose that the following three conditions are satisfied:

(i) $\gamma(E) \geq r_{k}(E)$,

(ii) $\gamma\left(f^{*} E\right) \geq r_{k}\left(f^{*} E\right)$,

(iii) $f$ is a local biholomorphism at $P \in X$.

Then $P \in W_{k}\left(f^{*} E\right)$ implies $f(P) \in W_{k}(E)$.

Proof. Put $Q=f(P)$. Since $f$ is a local biholomorphism at $P$, the map $\psi$ in diagram (*) is onto. Now, if $Q \notin W_{k}(E)$, then $\alpha$ is onto; hence $\beta$ must be onto and $P \notin W_{k}\left(f^{*} E\right)$.

(3.2) Corollary. Let $X$ (resp. $X^{\prime}$ ) be a compact Riemann surface of genus $g$ (resp. $g^{\prime}$ ) and canonical bundle $T$ (resp. $T^{\prime}$ ). Let $f: X \rightarrow X^{\prime}$ be a smooth cover. Then $f\left(W_{k}(T)\right) \subseteq W_{k}\left(T^{\prime}\right)$ for $k \leq g^{\prime}-1$. In particular, if $X$ is hyperelliptic, then $X^{\prime}$ is hyperelliptic.

Proof. Since $f$ is unramified, $f^{*} T^{\prime}=T$. The first statement is then an easy consequence of the theorem. The second statement uses the fact that $X$ is hyperelliptic if and only if $X$ has a hyperelliptic Weierstrass point $\left(\left[3\right.\right.$, p. 228]), which is equivalent to $W_{1}(T)$ being nonempty.

(3.3) Remark. As has been pointed out to me by R. D. M. Accola and A. Nobile, 3.2 may be proved by elementary methods and without the assumption that $f$ is unramified. The idea is as follows. Let $L$ (resp. $L^{\prime}$ ) denote the field of meromorphic functions on $X$ (resp. on $X^{\prime}$ ). Then if $P \in W_{k}(T)$ and $h \in L$ has a pole of order at most $k+1$ at $P$ and no other poles, then $\operatorname{Tr}_{L / L^{\prime}}(h)$, the trace of $h$, will be an element of $L^{\prime}$ with pole of order at most $k+1$ at $f(P)$ and no other poles.

\section{REFERENCES}

1. A. Altman and S. Kleiman, Introduction to Grothendieck duality theory, Lecture Notes in Mathematics No. 146 (Springer-Verlag, 1970). 1973).

2. M. Golubitsky and V. Guillemin, Stable mappings and their singularities (Springer-Verlag, 1966).

3. R. C. Gunning, Lectures on Riemann surfaces (Princeton University Press, Princeton, N.J., 1966).

4. F. Hirzebruch, Topological methods in algebraic geometry, Third edition (Springer-Verlag,

5. R. F. Lax, Weierstrass points of products of Riemann surfaces, Pacific J. Math. 66 (1976), 191-194. 
6. R. H. Ogawa, On the points of Weierstrass in dimension greater than one, Trans. Amer. Math. Soc. 184 (1973), 401-417.

7. H. E. Rauch and H. M. Farkas, Theta functions with applications to Riemann surfaces (Williams and Wilkins, Baltimore, 1974).

Louisiana State University,

Baton Rouge, Louisiana 70803, U.S.A. 